\title{
Projecting demand for critical care beds during COVID-19 outbreaks in Canada
}

\author{
Affan Shoukat PhD, Chad R. Wells PhD, Joanne M. Langley MD, Burton H. Singer PhD, Alison P. Galvani PhD, \\ Seyed M. Moghadas PhD
}

- Cite as: CMAJ 2020 May 11;192:E489-96. doi: 10.1503/cmaj.200457; early-released April 8, 2020

See related editorial at www.cmaj.ca/lookup/doi/10.1503/cmaj.200606

\begin{abstract}
BACKGROUND: Increasing numbers of coronavirus disease 2019 (COVID-19) cases in Canada may create substantial demand for hospital admission and critical care. We evaluated the extent to which self-isolation of mildly ill people delays the peak of outbreaks and reduces the need for this care in each Canadian province.
\end{abstract}

METHODS: We developed a computational model and simulated scenarios for COVID-19 outbreaks within each province. Using estimates of COVID-19 characteristics, we projected the hospital and intensive care unit (ICU) bed requirements without self-isolation, assuming an average number of 2.5 secondary cases, and compared scenarios in which different proportions of mildly ill people practised selfisolation 24 hours after symptom onset.

RESULTS: Without self-isolation, the peak of outbreaks would occur in the first half of June, and an average of 569 ICU bed days per 10000 population would be needed. When $20 \%$ of cases practised self-isolation, the peak was delayed by 2-4 weeks, and ICU bed requirement was reduced by $23.5 \%$ compared with no self-isolation.
Increasing self-isolation to $40 \%$ reduced ICU use by $53.6 \%$ and delayed the peak of infection by an additional 2-4 weeks. Assuming current ICU bed occupancy rates above $80 \%$ and self-isolation of $40 \%$, demand would still exceed available (unoccupied) ICU bed capacity.

INTERPRETATION: At the peak of COVID19 outbreaks, the need for ICU beds will exceed the total number of ICU beds even with self-isolation at $40 \%$. Our results show the coming challenge for the health care system in Canada and the potential role of self-isolation in reducing demand for hospital-based and ICU care. ince the identification of severe acute respiratory syndrome coronavirus 2 (SARS-CoV-2) in December 2019 in China, coronavirus disease 2019 (COVID-19) has caused more than 850000 confirmed cases across 180 countries, including Canada. ${ }^{1}$ As of Mar. 31, 2020, there were 8591 total confirmed cases across Canada, including cases caused by local transmission in several communities. The current approach to public health at the federal level aims at decreasing disease transmission through social-distancing strategies and self-isolation of people who are potentially exposed to disease or those with symptoms who do not need hospital care.

Initial outbreaks and experience in affected countries show a substantial surge in demand for hospital and critical care of COVID-19 patients. ${ }^{2-7}$ Public health efforts to mitigate this surge focus on voluntary social-distancing behavioural changes such as self-isolation, reduced mass gatherings and better hygiene practices. Until vaccines or therapies are available, these effective nonpharmaceutical interventions are intended to decrease the rate of rise of new infections so that new cases occur over a more prolonged period, often referred to as "flattening the curve." This approach is to avoid overwhelming health care systems, ${ }^{7}$ which have finite capacity. Although these measures contribute to overall pandemic response strategies, they are not expected to contain COVID-19 outbreaks.

A primary concern in public health planning is the use of health care resources for treatment of patients needing critical care. ${ }^{8}$ For example, in Winnipeg, Manitoba, critical care was severely challenged during the initial peak of the influenza $A$ (H1N1) virus pandemic in June 2009, as intensive care units (ICUs) were at full capacity. ${ }^{9,10}$ At the peak of the second wave, usage of invasive ventilators was $14 \%$ greater than average, with $50 \%$ of this increase being caused by cases of H1N1-associated illness. ${ }^{8}$ With Canadian ICU bed occupancy already close to capacity before a public health crisis ${ }^{11}$ and varying widely across jurisdictions, ${ }^{12}$ it is imperative to ascertain the extent to which these resources will be required in a COVID-19 outbreak scenario.

Our objective was to project the timing and magnitude of demand for critical care beds at the peak of COVID-19 outbreaks in Canadian provinces. 


\section{Methods}

We developed an age-structured, agent-based model and parameterized it with the age distribution of the population in each province as well as early estimates of COVID-19-specific characteristics. In scenario analyses, we considered self-isolation by mildly symptomatic cases as a control measure and evaluated its effect on the outbreak trajectory and the need for critical care. We simulated the model under the scenarios in which the average number of secondary infections, referred to as the basic reproduction number $R_{0}$, was 2 and 2.5..$^{13,14}$

\section{Model structure}

We developed a stochastic, age-stratified, agent-based computational model for the transmission dynamics of COVID-19. This computational model simulates autonomous agents (representing individuals in a human population) and their interactions within a constrained virtual environment. We accounted for the various epidemiologic statuses of individuals, including susceptible; infected and incubating; infectious and symptomatic with mild, severe or critical illness; recovered; and dead (Figure 1). For each province, we stratified the population into 5 age groups $-0-4$ years, 5-19 years, 20-49 years, 50-64 years and $\geq 65$ years - based on demographic data from the latest Canadian census. ${ }^{15}$ We simulated disease transmission within and among these age groups by considering an empirically determined contact network. ${ }^{16}$ Specifically, we sampled the daily number of contacts for each individual from an age-specific negative-binomial distribution (Appendix 1, Table A1, available at www.cmaj.ca/lookup/suppl/doi:10.1503/cmaj.200457/-/DC1), based on a contact matrix for urban and densely populated regions.

\section{Disease dynamics}

We implemented disease transmission probabilistically for contacts between susceptible and symptomatic individuals, with a daily transmission probability determined through model calibration (Table 1). After infection, the newly infected individuals entered an incubation period for an average of 5.2 days. ${ }^{13,18}$ After the incubation period, individuals entered a symptomatic phase, with an average communicability period of 4.6 days. We calculated this period using an estimated average of 7.5 days for the serial interval (defined as the duration between the onset of symptoms in an index case to the onset of symptoms in a secondary case)..$^{13}$ Symptomatic cases had an age-dependent probability of developing mild, severe or critical illness. ${ }^{7,19}$ We assumed that the relative infectivity of individuals with mild illness compared with those with severe illness was reduced by $50 \%{ }^{17}$

\section{Infection outcomes}

We assumed that mild cases would recover without the need for hospital admission, but may practise self-isolation. Hospital and ICU admissions were considered for severe and critically ill individuals. For those who were admitted to hospital, the average time from symptom onset to admission was uniformly sampled in the range of 2 to 5 days. ${ }^{7,20}$ Patients in hospital were assumed to be effectively isolated by infection prevention and control measures and to no longer spread infection. Patients admitted to the hospital occupied a non-ICU bed for an average of 11.5 days before recovery. ${ }^{7,20}$ Further, patients in hospital had an agedependent probability of being admitted to the ICU. For these patients, the average length of ICU bed occupancy was 14.4 days before recovery. ${ }^{7}$

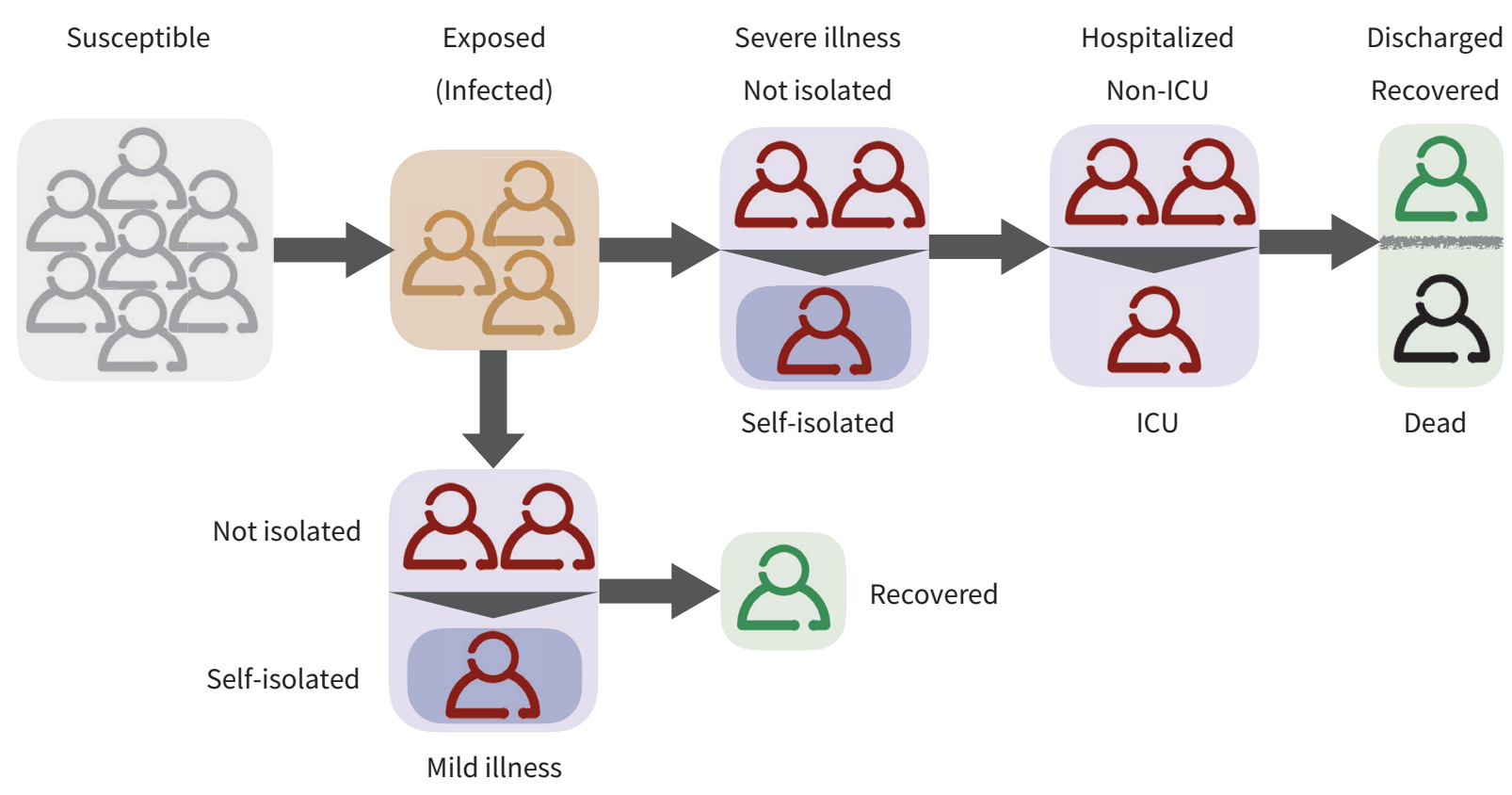

Figure 1: Schematic diagram of the model for natural history of the disease and implementation of self-isolation and hospital admission. Note: ICU = intensive care unit. 


\section{Self-isolation}

Symptomatic individuals who practised self-isolation were assumed to stay isolated until the end of their infectious period. In our model, the effect of self-isolation was implemented by reducing the number of daily contacts to a maximum of 3 . In the absence of data quantifying the proportion of symptomatic individuals who practise self-isolation, we varied this proportion in the range $0 \%-80 \%, 24$ hours after symptom onset. For severe cases, we assumed $80 \%$ would self-isolate within 24 hours after symptom onset before hospital admission. Our assumption is based on previous estimates, ${ }^{22}$ as well as on the perceived seriousness of COVID-19 infection and emphasis on social distancing and isolation by public health agencies.

\section{Critical care capacity}

A 2015 national cross-sectional study estimated a total of 3170 ICU beds capable of invasive ventilation across Canada. ${ }^{12}$ To project the hospital and ICU bed demand at the peak of outbreaks, we calculated the number of existing ICU beds in each province and estimated the unoccupied ICU beds that could be used for treatment of COVID-19 patients, assuming a conservative occupancy rate of $80 \%$ for existing beds. ${ }^{23}$

\section{Model implementation}

To project the hospital and ICU bed demand for each province, we calibrated the model to a reproduction number $R_{0}=2.5$ for the base-case scenario, as well as an alternative scenario of $R_{0}=$ 2.0. ${ }^{13,14}$ Model parameter values were informed by latest estimates of COVID-19 characteristics and were sampled from relevant distributions (Table 1). The model was computationally implemented in Julia language. ${ }^{24}$ We seeded all simulations with 5 initial symptomatic cases within a population of 10000 individuals, with the start date of Mar. 1, 2020. We averaged the results for daily incidence of infection and hospital admission over 500 independent simulations. We estimated the average number of ICU bed days needed over the course of COVID-19 outbreaks using the mean prevalence of all simulations, counting the cumulative number of days that ICU beds were occupied by patients during the outbreak. The computational system is available at https://github.com/affans/covid19abm.jl.

Table 1: Description of parameters and their values or ranges for each age group

\begin{tabular}{|c|c|c|c|c|c|}
\hline \multirow[b]{2}{*}{ Description } & \multicolumn{4}{|c|}{ Age group } & \multirow[b]{2}{*}{ References } \\
\hline & $0-19 \mathrm{yr}$ & $20-49 \mathrm{yr}$ & $50-64$ yr & $\geq 65 \mathrm{yr}$ & \\
\hline \multicolumn{6}{|l|}{$\begin{array}{l}\text { Transmission probability per contact, } \\
\text { calibrated to } R_{0}=2,2.5\end{array}$} \\
\hline$R_{0}=2$ & 0.0356 & 0.0356 & 0.0356 & 0.0356 & \multirow[t]{2}{*}{ Li et al., ${ }^{13} \mathrm{Wu}$ et al. ${ }^{14}$} \\
\hline$R_{0}=2.5$ & 0.0455 & 0.0455 & 0.0455 & 0.0455 & \\
\hline $\begin{array}{l}\text { Relative infectivity of mild illness compared } \\
\text { with severe and critical illness }\end{array}$ & 0.5 & 0.5 & 0.5 & 0.5 & Li et al. ${ }^{17}$ \\
\hline Average duration of incubation period, $d$ & $\begin{array}{c}\log N \\
(5.2,0.1)\end{array}$ & $\begin{array}{c}\log N \\
(5.2,0.1)\end{array}$ & $\begin{array}{c}\log N \\
(5.2,0.1)\end{array}$ & $\begin{array}{c}\operatorname{LogN} \\
(5.2,0.1)\end{array}$ & Li et al., ${ }^{13}$ Lauer et al. ${ }^{18}$ \\
\hline $\begin{array}{l}\text { Proportion of cases who exhibit mild symptoms } \\
\text { (based on reported cases in different age groups) }\end{array}$ & 0.80 & 0.80 & 0.40 & 0.20 & $\begin{array}{l}\text { Moghadas et al., } \\
\qquad \text { WHO }^{19}\end{array}$ \\
\hline Average infectious period, $d$ & 4.6 & 4.6 & 4.6 & 4.6 & Estimated \\
\hline $\begin{array}{l}\text { Probability of self-isolation after symptom } \\
\text { onset for severe and critical cases }\end{array}$ & 0.80 & 0.80 & 0.80 & 0.80 & Assumed \\
\hline $\begin{array}{l}\text { Probability of self-isolation after symptom } \\
\text { onset for mild cases }\end{array}$ & $0-0.8$ & $0-0.8$ & $0-0.80$ & $0-0.80$ & Varied \\
\hline Time from symptom onset to self-isolation, $d$ & 1 & 1 & 1 & 1 & Assumed \\
\hline $\begin{array}{l}\text { Average time from symptom onset to hospital } \\
\text { admission, } d\end{array}$ & $\begin{array}{l}\text { Unif } \\
(2,5)\end{array}$ & $\begin{array}{l}\text { Unif } \\
(2,5)\end{array}$ & $\begin{array}{l}\text { Unif } \\
(2,5)\end{array}$ & $\begin{array}{l}\text { Unif } \\
(2,5)\end{array}$ & $\begin{array}{l}\text { Moghadas et al., } \\
\text { Sanche et al. }\end{array}$ \\
\hline $\begin{array}{l}\text { Proportion of symptomatic patients with } \\
\text { severe and critical illness requiring hospital } \\
\text { care or ICU }\end{array}$ & $\begin{array}{c}\text { Unif } \\
(0.02,0.03)\end{array}$ & $\begin{array}{c}\text { Unif } \\
(0.28,0.34)\end{array}$ & $\begin{array}{l}\text { Unif } \\
(0.28,0.34)\end{array}$ & $\begin{array}{c}\text { Unif } \\
(0.60,0.68)\end{array}$ & $\begin{array}{l}\text { Estimated from WHO, }{ }^{19} \\
\text { MIDAS }{ }^{21}\end{array}$ \\
\hline $\begin{array}{l}\text { Proportion of cases admitted to hospital who } \\
\text { require ICU }\end{array}$ & $\begin{array}{c}\text { Unif } \\
(0.01,0.013)\end{array}$ & $\begin{array}{c}\text { Unif } \\
(0.03,0.05)\end{array}$ & $\begin{array}{l}\text { Unif } \\
(0.05,0.2)\end{array}$ & $\begin{array}{l}\text { Unif } \\
(0.05,0.15)\end{array}$ & Estimated from MIDAS ${ }^{21}$ \\
\hline Length of hospital stay before recovery, $d$ & \multicolumn{4}{|c|}{ Gamma $(4.5,2.75)$ truncated between 8 and 17} & $\begin{array}{l}\text { Moghadas et al.., } \\
\text { Sanche et al. }{ }^{20}\end{array}$ \\
\hline Length of ICU stay before recovery, $d$ & \multicolumn{4}{|c|}{ Gamma $(4.5,2.75)$ truncated between 10 and 19} & $\begin{array}{l}\text { Moghadas et al., } \\
\text { Sanche et al. }{ }^{20}\end{array}$ \\
\hline
\end{tabular}

Note: $\operatorname{Gamma}(a, b)=$ gamma distribution with shape parameter a and scale parameter $b, \mathrm{ICU}=$ intensive care unit, $\operatorname{LogN}(a, b)=\log$ normal distribution with shape parameter $a$ and scale parameter $b$, Unif $(a, b)=$ uniform distribution with mean $a$ and standard deviation $b$. 


\section{Ethics approval}

This research was based on publicly available data and therefore did not require ethics approval.

\section{Results}

The number of existing ICU beds in each province ranged from 0.63 to 1.85 per 10000 population. Assuming an existing occupancy rate of $80 \%$, we estimated that unoccupied ICU beds available for treatment of COVID-19 patients would range across provinces from 0.13 to 0.37 per 10000 population (Table 2).

In the base-case scenario of $R_{0}=2.5$ without self-isolation by mild cases, we projected that each province would need an average of 569 ICU bed days (range 548-587) per 10000 population over the course of COVID-19 outbreaks across all provinces (Figure 2). In this scenario, the outbreaks lasted for 36 weeks (Appendix 1, Figures A1A4). Alberta was projected to have the largest utilization of ICU (mean 587 bed days per 10000 population; median 589; interquartile range [IQR] 525-657), whereas Prince Edward Island would have the lowest (mean 561 bed days per 10000 population; median 566; IQR 497-623) (Figure 2). We estimated that an average of 41 ICU beds per 10000 population would be required for the duration of outbreaks in each province. This average corresponds to the cumulative number of patients requiring ICU admission during the outbreak. We projected the peak of infection to occur in the first half of June (Figure 3), followed by the peak of prevalence for admissions to hospital in mid- to late June (Appendix 1, Appendix Figures A1-A4). Although differences across provinces were small, we projected that Manitoba and British Columbia would have the largest need for ICU beds per 10000 population at the peak, given the available (i.e., unoccupied) ICU beds (Table 2).

Table 2: Projected peak capacity requirements for ICU and non-ICU hospital beds per 10000 population, across a range of self-isolation scenarios by mildly symptomatic cases in different provinces*

\section{Hospital bed requirements at the peak}

\section{Variables}

Self-isolation, \%

Province

British Columbia

Mean: 80.2

Median: 83

(IQR 72-92)

Alberta

Mean: 81

Median: 82

(IQR 73-93)

Saskatchewan

Mean: 81.1

Median: 83

(IQR 72-92)

Manitoba

Mean: 81

Median: 82

(IQR 73-95)

Ontario

Mean: 80.7

Median: 83

(IQR 72-92)

Quebec

Mean: 81.1

Median: 83

(IQR 72-92)

New Brunswick

Mean: 80.2

Median: 82

(IQR 72-93)

Prince Edward

Mean: 81.1

Island

Nova Scotia

Median: 83

(IQR 72-92)

Mean: 80.7

Median: 83

(IQR 72-92)

Newfoundland

Mean: 81

and Labrador
Non-ICU beds

20

40

Mean: 46.6

Median: 48

(IQR 38-55)

Mean: 46.9

Median: 48

(IQR 39-56)

Mean: 47.0

Median: 48

(IQR 39-56)

Mean: 46.9

Median: 48

(IQR 39-56)

Mean: 47.0

Median: 48

(IQR 39-65)

Mean: 47.0

Median: 48

(IQR 40-56)

Mean: 46.6

Median: 48

(IQR 39-55)

Mean: 47.0

Median: 48

(IQR 40-56)

Mean: 47.0

Median: 48

(IQR 38-56)

Mean: 46.7

Median: 47

(IQR 40-56)
Mean: 18.6

Median: 19

(IQR 12-25)

Mean: 18.9

Median: 19

(IQR 14-25)

Mean: 18.9

Median: 19

(IQR 13-25)

Mean: 18.8

Median: 19

(IQR 13-25)

Mean: 18.9

Median: 19

(IQR 14-25)

Mean: 18.3

Median: 19

(IQR 11-26)

Mean: 18.7

Median: 19

(IQR 12-25)

Mean: 18.3

Median: 19

(IQR 11-26)

Mean: 18.4

Median: 19

(IQR 11-25)

Mean: 18.9

Median: 19

(IQR 12-25)
ICU beds

ICU beds per
10000
population

20

40

\begin{tabular}{cl} 
Mean: 7.5 & Mean: 4.2 \\
Median: 7 & Median: \\
(IQR 6-10) & (IQR 2-6) \\
\hline Mean: 7.5 & Mean: 4.3 \\
Median: 7 & Median: 4 \\
(IQR 6-9.5) & (IQR 3-6) \\
Mean: 7.6 & Mean: 4.3 \\
Median: 7 & Median: \\
(IQR 5-10) & (IQR 3-6) \\
\hline Mean: 7.6 & Mean: 4.2 \\
Median: 7 & Median: \\
(IQR 5-10) & (IQR 2-6) \\
Mean: 7.5 & Mean: 4.2 \\
Median: 7 & Median: 4 \\
(IQR 5-9.5) & (IQR 2-6) \\
\hline Mean: 7.5 & Mean: 4.2 \\
Median: 7 & Median: \\
(IQR 5-9) & (IQR 2-6) \\
\hline Mean: 7.6 & Mean: 4.2 \\
Median: 8 & Median:4 \\
(IQR 5.5-9) & (IQR 2-4) \\
Mean: 7.5 & Mean: 4.2 \\
Median: 7 & Median: 4 \\
(IQR 5-9) & (IQR 2-6) \\
Mean: 7.5 & Mean: 4.2 \\
Median: 7 & Median: 4 \\
(IQR 5-9) & (IQR 2-6) \\
\hline Mean: 7.6 & Mean: 4.2 \\
Median: 7 & Median:4 \\
(IQR 5-10) & (IQR 3-6) \\
& \\
\hline
\end{tabular}

Mean: 1.6

Median: 1

(IQR 0-3)

Mean: 1.6

Median: 1

(IQR 0-2)

Mean: 1.8

Median: 1

(IQR 0.5-3)

Mean: 1.8

Median: 1

(IQR 0-3)

Mean: 1.7

Median: 1

(IQR 1-3)

Mean: 1.7

Median: 1

(IQR 1-2)

Mean: 1.7

Median: 1

(IQR 1-3)

Mean: 1.6

Median: 1

(IQR 0-3)

Mean: 1.7

Median: 1

(IQR 1-3)

Mean: 1.7

Median: 2

(IQR 1-2)
Existing: 0.63

Unoccupied: 0.13

Existing: 0.68

Unoccupied: 0.14

Existing: 0.93

Unoccupied: 0.19

Existing: 0.69

Unoccupied: 0.14

Existing: 0.79

Unoccupied: 0.16

Existing: 1.05

Unoccupied: 0.21

Existing: 1.36

Unoccupied: 0.27

Existing: 1.84

Unoccupied: 0.24

Existing: 1.48

Unoccupied: 0.30

Existing: 1.85

Unoccupied: 0.37

Note: COVID-19 = coronavirus disease $19, \mathrm{ICU}=$ intensive care unit, IQR = interquartile range.

${ }^{*}$ Reported estimates are mean and IQR. We assumed an occupancy rate of $80 \%$ for existing ICU beds to calculate the number of ICU beds available for treatment of COVID-19 patients. 


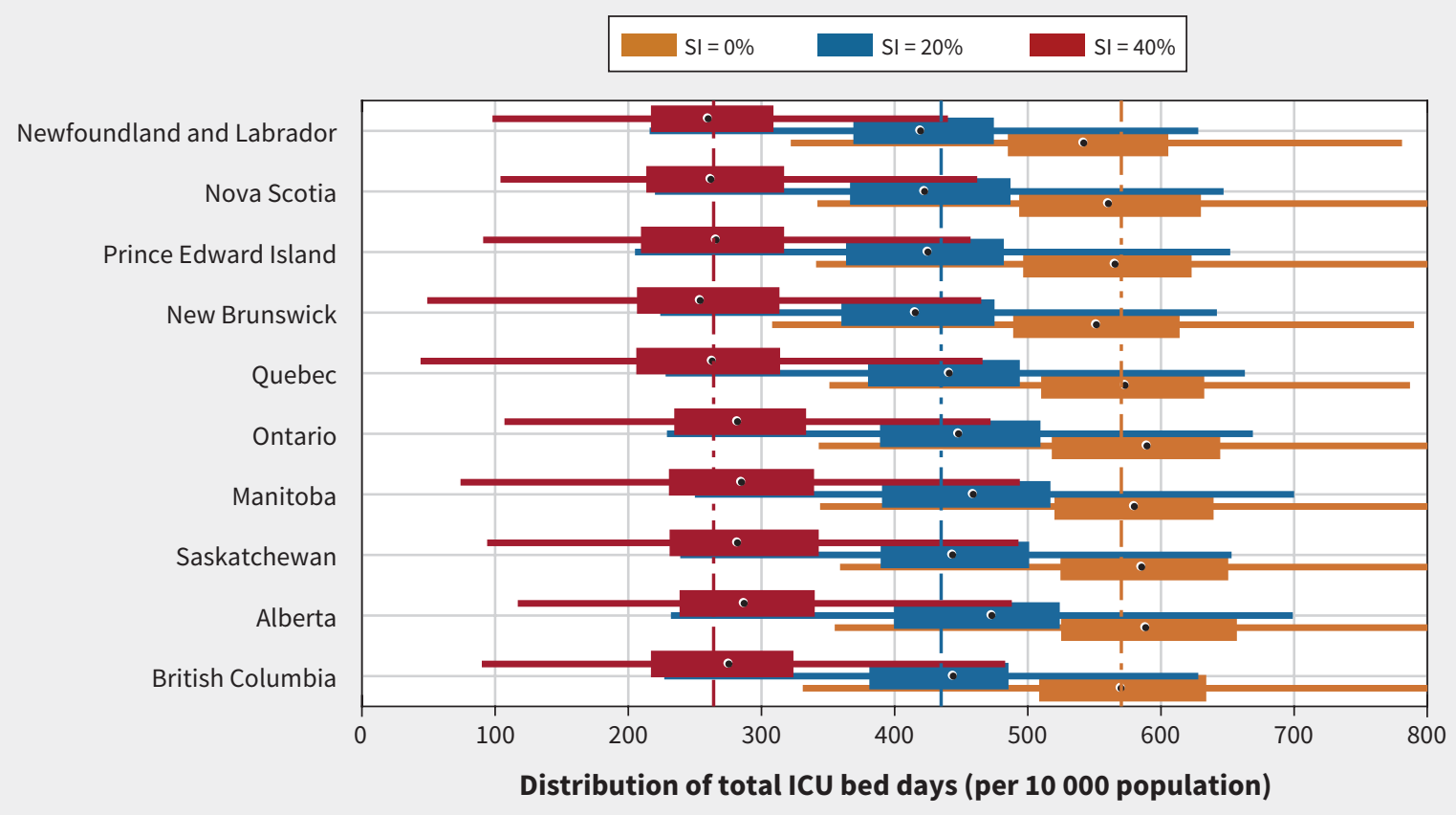

Figure 2: Distribution of the cumulative intensive care unit (ICU) bed days per 10000 population during the coronavirus disease 2019 (COVID-19) outbreaks in different provinces. Box plots correspond to the level of self-isolation (SI) among mild symptomatic cases: $\mathrm{SI}=0 \%$ (orange), $\mathrm{SI}=20 \%$ (blue) and $\mathrm{SI}=40 \%$ (red). Circles on box plots indicate medians; bars indicate interquartile range (IQR); horizontal lines are extended range from minimum (25th percentile - 1.5 IQR) to maximum (75th percentile $+1.5 \mathrm{IQR}$ ). Vertical dashed lines correspond to the average ICU bed days in all provinces without self-isolation (orange line $=569$ ), $20 \%$ self-isolation (blue line $=435$ ) and $40 \%$ self-isolation (red line $=264$ ). These distributions are based on 500 independent simulations in each scenario.

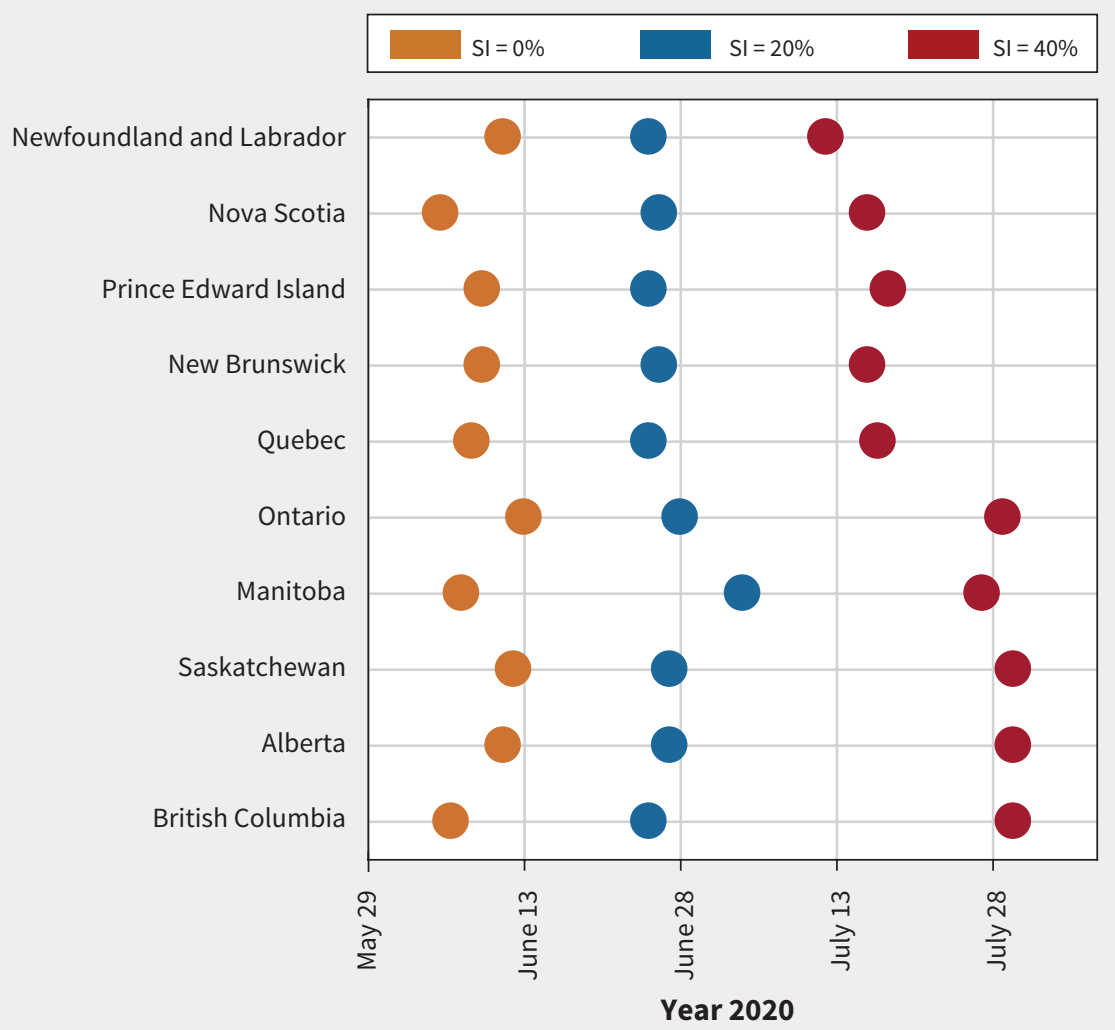

Figure 3: Projected week of the outbreak peak (based on the highest daily incidence of new infections) in different provinces. Coloured circles correspond to the level of self-isolation (SI) among mild symptomatic cases. 
We next evaluated scenarios in which self-isolation by symptomatic individuals with mild illness reduces hospital admissions and delays the outbreak peak. When $R_{0}=2.5$ and selfisolation was practised by $20 \%$ of symptomatic individuals with mild illness and $80 \%$ of symptomatic individuals with severe illness within 24 hours after symptom onset, we observed a decline of $23.6 \%$ in the overall use of ICU beds to 435 (range 414 to 461 ) bed days per 10000 population (Figure 2). The average number of ICU beds per 10000 population required for the duration of outbreaks was estimated at 31 in each province, a reduction of $24.4 \%$ compared with no self-isolation. Furthermore, the peak of infection during outbreaks was delayed by 4 weeks in Manitoba and about 2-3 weeks in other provinces (Figure 3), while the magnitude of peaks reduced (Appendix 1, Figures A1A4). This level of isolation reduced the need for non-ICU and ICU beds at the peak by $42 \%$ and $43 \%$, respectively, across all provinces (Table 2).

Increasing the proportion of cases who self-isolate to $40 \%$ further reduced the overall ICU bed days per 10000 population by $53.6 \%$ to 264 (range 250-279) (Figure 2), and delayed the peak of outbreaks by up to 8 weeks (Figure 3), compared with no selfisolation. In this scenario, the earliest outbreak peak among all provinces was projected to occur in mid-July. We also observed earlier peaks in the eastern provinces compared with Ontario and western provinces (Figure 3). At the peak of outbreaks, we projected a reduction of $78 \%$ in the need for non-ICU beds and $79 \%$ in the need for ICU beds, with an outbreak duration 12 weeks longer than with no self-isolation. We estimated that an average of 19 ICU beds per 10000 population would be required for the duration of outbreaks in each province, a reduction of $53.7 \%$ compared with no self-isolation. The results for higher proportion of self-isolation (60\% and $80 \%)$ are provided in Appendix 1, Figures A20-A29.

Extending our analysis to a lower reproduction number at $R_{0}=$ 2.0, we observed qualitatively similar results, although the proportional impact of self-isolation on reducing the number of ICU beds needed at the peak of outbreaks was substantially higher (Appendix 1). In an analysis in which self-isolation was assumed to start at 48 hours after symptom onset rather than 24 hours, results show a higher peak of outbreaks as well as increased need for ICU bed days compared with self-isolation 24 hours after symptom onset (Appendix 1, Figures A20-A29).

\section{Interpretation}

In the absence of a vaccine or evidence-based disease-altering therapies, self-isolation of symptomatic cases can decelerate the rate of disease spread and reduce the surge capacity needed to manage hospital admissions for COVID-19. Under a plausible scenario of $R_{0}=2.5$, our findings indicate that even $40 \%$ selfisolation of symptomatic cases 24 hours after symptom onset is still inadequate to bring the need for ICU beds at the peak to below the existing capacity in all provinces. At the peak of COVID-19 outbreaks, we projected that the required ICU beds per 10000 population (on average) can be as high as 2.6 times the existing number of beds. A conservative hospital-bed occupancy rate of $80 \%$ suggests that the need for critical care may be as much as 13 times higher than the available (unoccupied) ICU beds at the peak of outbreak even with $40 \%$ self-isolation.

Our results show that higher rates of self-isolation are needed to mitigate the increased strain on the health care systems at the provincial level. Should resources allow for an increase in COVID19 testing, a higher fraction of mildly symptomatic cases would be identified, which could lead to a higher percentage of the population practising self-isolation. If infection confers immunity, a testing strategy could also permit recovered people to return to normal activity.

\section{Limitations}

Our results should be interpreted in the context of model assumptions. As the outbreak progresses locally, control measures will be adapted and possibly expanded, which may change the trajectory of the outbreaks and would therefore affect our results. We have not explicitly modelled other social-distancing measures, but their effect can be largely incorporated in the reduction of disease transmission by varying degrees of selfisolation, which could both delay and lower the peak of outbreaks and therefore the demand for critical care.

Furthermore, refined spatial dynamics within each province could delay the timing of peak capacity at a regional level and the peak of the surge for hospital care. For example, social (physical) distancing of well persons could further reduce transmission and, ultimately, the burden of disease. Further, the settings in which individuals self-isolate, such as urban or rural communities, could alter the effectiveness of self-isolation, particularly in the context of more vulnerable subpopulations, such as the homeless and marginally housed, those with precarious employment, and rural and isolated communities.

Jurisdictions may also vary with respect to the kind of local measures that are implemented and how well the population adheres to public health guidance. Our results are presented at a provincial level, aggregating any regional spatial heterogeneity regarding population characteristics. Despite the expected variability at a regional or more local scale, the qualitative findings across all provinces can be useful for informing localized strategies within provinces.

Our projections for the effect of self-isolation rely on the assumption that all infected cases manifest symptomatic disease in either mild or severe form. Given the possibility of asymptomatic and presymptomatic transmission, ${ }^{25}$ the effect of selfisolation by symptomatic cases may be reduced. Although we have not explicitly modelled these stages of illness, delaying the start of self-isolation (e.g., 48 hours rather than 24 hours) after symptom onset in our model could account for the effect of potential transmission in the presymptomatic stage.

We also assumed that patients in hospital are effectively isolated and no longer contribute to infection spread to others (e.g., health care workers). Given strict adherence to infection prevention and control measures in treatment of COVID-19 patients, we think this is a reasonable assumption in the acute care hospital-based setting in Canada. However, COVID-19 has 
been reported in health care workers in Canada and elsewhere. Where community transmission is established, it is more likely that these cases are community acquired rather than a result of poor adherence to infection prevention and control by health care workers during the performance of their duties. Factors such as shortages of personal protective equipment, crowding, extreme fatigue or stress that could lead to errors in adhering to best practice would increase the risk that health care personnel could acquire infection at work and transmit it in the health care setting and to their home and community contacts until they are identified as unwell. This effect would be minimized by adherence to recommendations for infection control, early recognition of illness in staff and the reduced likelihood that health care workers will be caring for non-COVID-19 patients as the outbreaks increase in magnitude. Although health care worker infection could reduce the effect of isolation in a hospital setting, accurate quantification of the combined effect of the aforementioned factors is not available but could be incorporated into future models as evidence accumulates.

Our model does not include seasonal factors, which may affect disease transmission with possible declining epidemic trend in the summer and resurgence of disease concomitant with influenza in the next season. The lack of quantification for these factors and incomplete understanding of the characteristics of COVID-19 at this point in time indicate the uncertainty regarding any quantitative projections in modelling efforts. However, as new information and data pertinent to COVID-19 become available, our model can be reparameterized to provide a more accurate projection on outbreak trajectories and the demand for critical care. Despite these considerations, our findings highlight the importance of social-distancing measures, and in particular selfisolation, on reducing the need for hospital and critical care during COVID-19 outbreaks.

\section{Conclusion}

Our results highlight the importance of self-isolation in reducing the burden on health care facilities during the expected COVID19 outbreaks that will occur in the coming months. The relatively low capacity of critical care in Canada - combined with hospital occupancy rates above $90 \%$ and, in many regions, close to or over the capacity in interpandemic periods ${ }^{23}$ - underscore the challenges for treatment of critically ill patients during and especially at the peak of outbreaks. The experience with the COVID-19 outbreak in countries that are already at or past their peak can inform Canadian planning. If the scenarios we describe are true, health care planning will move to crisis management in Canada, but at different times across the country. This may allow some redistribution of human or material resources across jurisdictions. While provinces and territories ramp up capacity for more ICU beds (human resources, ventilators, beds, personal protective equipment, infection prevention and control support), these estimates can inform the timing and quantity of the expected need. Laboratory confirmation of the degree of community spread will aid in predicting the shape of the local outbreaks. Provincial and federal governments have been taking the initiative to rapidly expand testing capacity, which will aid in disease surveillance. ${ }^{26-29}$ Experience in other countries also suggests that early transfer of severe cases to the ICU can help to stabilize patients' conditions before patients exhibit respiratory failure. ${ }^{30,31}$ Best practices for care of critically ill patients is evolving rapidly.

\section{References}

1. Coronavirus COVID-19 global cases by the Center for Systems Science and Engineering (CSSE) at Johns Hopkins University (JHU). Johns Hopkins Coronavirus Resource Center. Available: www.arcgis.com/apps/opsdashboard/index. html\#/bda7594740fd40299423467b48e9ecf6 (accessed 2020 Mar. 19).

2. Ñamendys-Silva SA. Respiratory support for patients with COVID-19 infection. Lancet Respir Med 2020 Mar. 5:pii: S2213-2600(20)30110-7 [Epub ahead of print]. doi: 10.1016/S2213-2600(20)30110-7.

3. Wang D, Hu B, Hu C, et al. Clinical characteristics of 138 hospitalized patients with 2019 novel coronavirus-infected pneumonia in Wuhan, China. JAMA 2020 Feb. 7 [Epub ahead of print]. doi: 10.1001/jama.2020.1585.

4. Yang $X, Y u Y, X u$ J, et al. Clinical course and outcomes of critically ill patients with SARS-CoV-2 pneumonia in Wuhan, China: a single-centered, retrospective, observational study. Lancet Respir Med 2020 Feb. 24:pii: S2213-2600(20)30079-5 [Epub ahead of print]. doi: 10.1016/S2213-2600(20)30079-5.

5. Huang C, Wang Y, Li X, et al. Clinical features of patients infected with 2019 novel coronavirus in Wuhan, China. Lancet 2020;395:497-506.

6. Guan W-j, Ni Z-y, Hu Y, et al. Clinical Characteristics of Coronavirus Disease 2019 in China. N Engl J Med 2020 Feb. 28 [Epub ahead of print]. doi: 10.1056/ NEJMoa2002032.

7. Moghadas SM, Shoukat A, Fitzpatrick MC, et al. Projecting hospital utilization during the COVID-19 outbreaks in the United States. Proc Natl Acad Sci U S A. 2020 Apr. 3 [Epub ahead of print]. doi: 10.1073/pnas.2004064117.

8. The impact of the H1N1 pandemic on Canadian hospitals. Ottawa: Canadian Institute for Health Information; 2010. Available: https://secure.cihi.ca/free_ products/H1N1_AIB_final_EN.pdf (accessed 2020 Mar. 11).

9. Kumar A, Zarychanski R, Pinto R, et al. Canadian Critical Care Trials Group H1N1 Collaborative. Critically ill patients with 2009 influenza $A(H 1 N 1)$ infection in Canada. JAMA 2009;302:1872-9.

10. Hota S, Fried E, Burry L, et al. Preparing your intensive care unit for the second wave of H1N1 and future surges. Crit Care Med 2010;38(Suppl):e110-9.

11. Eggertson L. Critical care doctors want escalated pandemic planning. CMAJ 2009;181:253-4.

12. Fowler RA, Abdelmalik P, Wood G, et al.; Canadian Critical Care Trials Group. Canadian ICU Capacity Group. Critical care capacity in Canada: results of a national cross-sectional study. Crit Care 2015;19:133.

13. Li Q, Guan X, Wu P, et al. Early transmission dynamics in Wuhan, China, of novel coronavirus-infected pneumonia. N Engl J Med 2020;382:1199-207.

14. Wu JT, Leung K, Leung GM. Nowcasting and forecasting the potential domestic and international spread of the 2019-nCoV outbreak originating in Wuhan, China: a modelling study. Lancet 2020;395:689-97.

15. Population and demography statistics. Ottawa: Statistics Canada; modified 2019 Mar. 19. Available: www.statcan.gc.ca/eng/subjects-start/population and_demography (accessed 2020 Mar. 20).

16. Mossong J, Hens N, Jit M, et al. Social contacts and mixing patterns relevant to the spread of infectious diseases. PLoS Med 2008;5:e74.

17. Li R, Pei S, Chen B, et al. Substantial undocumented infection facilitates the rapid dissemination of novel coronavirus (SARS-CoV2). Science 2020 Mar. 16 pii: eabb3221 [Epub ahead of print]. doi: 10.1126/science.abb3221.

18. Lauer SA, Grantz KH, Bi Q, et al. The incubation period of coronavirus disease 2019 (COVID-19) from publicly reported confirmed cases: estimation and application. Ann Intern Med 2020 Mar. 10 [Epub ahead of print]. doi: 10.7326/ M20-0504

19. Report of the WHO-China joint mission on coronavirus disease 2019 (COVID-19). Geneva: World Health Organization; 2020 Feb. 16-24. Available: www.who.int/docs /default-source/coronaviruse/who-china-joint-mission-on-covid-19-final-report.pdf (accessed 2020 Mar. 9). 
20. Sanche S, Lin YT, Xu C, et al. The novel coronavirus, 2019-nCoV, is highly contagious and more infectious than initially estimated. medRxiv 2020 Feb. 11 [Epub ahead of print]. doi: 10.1101/2020.02.07.20021154.

21. MIDAS Network. MIDAS 2019 novel coronavirus repository. San Francisco: GitHub. Available: https://github.com/midas-network/COVID-19 (accessed 2020 Feb. 27).

22. Loustalot F, Silk BJ, Gaither A, et al. Household transmission of 2009 Pandemic influenza $A(\mathrm{H} 1 \mathrm{~N} 1)$ and nonpharmaceutical interventions among households of high school students in San Antonio, Texas. Clin Infect Dis 2011;52(Suppl 1):S146-53.

23. OECD. Hospital beds. In: Health at a Glance 2017: OECD Indicators. OECD (Organisation for Economic Co-operation and Development) Publishing, Paris, France; 2017:172-3.

24. Bezanson J, Edelman A, Karpinski S, et al. Julia: a fresh approach to numerical computing. SIAM Rev 2017;59:65-98.

25. Du Z, Xu X, Wu Y, et al. Serial interval of COVID-19 from publicly reported confirmed cases. Emerg Infect Dis 2020 Mar. 19 [Epub ahead of print]. doi: 10.3201/ eid2606.200357.
26. Province announces new measures to fight the coronavirus. 104.7 Heart FM 2020 Mar. 12. Available: www.heartfm.ca/news/local-news/province -announces-new-measures-to-fight-the-coronavirus/ (accessed 2020 Mar. 19).

27. Jones RP, Tunney C. Government expanding COVID-19 testing capacity and purchase of medical equipment, says federal health minister. CBC News 2020 Mar. 18, updated 2020 Mar. 19 2020. Available: www.cbc.ca/news/politics/coronavirus -test-capacity-medical-supplies-1.5500662 (accessed 2020 Mar. 19).

28. Wong J. Coronavirus: Alberta plans for expanded testing capacity and assessment centres. Global News 2020 Mar. 7. Available: https://globalnews.ca/news/6645687/ alberta-coronavirus-testing-assessment-centres/ (accessed 2020 Mar. 19).

29. Zussman RBC. premier outlines province's COVID-19 response plan. Global News 2020 Mar. 6. Available: www.//globalnews.ca/news/6641644/b-c-coronavirus -covid-19-update/ (accessed 2020 Mar. 19).

30. Murthy S, Gomersall CD, Fowler RA. Care for critically ill patients with COVID-19. JAMA 2020 Mar. 11 [Epub ahead of print]. doi: 10.1001/jama.2020.3633.

31. Liew MF, Siow WT, MacLaren G, et al. Preparing for COVID-19: early experience from an intensive care unit in Singapore. Crit Care 2020;24:83.
Competing interests: Joanne Langley reports that Dalhousie University has received payment for conduct of vaccine studies from Sanofi, GlaxoSmithKline, Merck, Janssen, VBI and Pfizer. Dr. Langley holds the Canadian Institutes of Health Research-GlaxoSmithKline Chair in Pediatric Vaccinology. No other competing interests were declared.

This article has been peer reviewed.

Affiliations: Center for Infectious Disease Modeling and Analysis (Shoukat, Wells, Galvani), Yale School of Public Health, New Haven, Conn.; Canadian Center for Vaccinology (Langley), Dalhousie University, IWK Health Centre and Nova Scotia Health Authority (Langley), Halifax, NS; Emerging Pathogens Institute (Singer), University of Florida, Gainesville, Fla.; Agent-Based Modelling Laboratory (Moghadas), York University, Toronto, Ont.

Contributors: Affan Shoukat, Alison Galvani, Joanne Langley and Seyed Moghadas contributed to the conception and design of the work. Affan Shoukat, Chad Wells, Joanne Langley and Seyed Moghadas contributed to the acquisition, analysis and interpretation of data. All of the authors drafted the manuscript, revised it critically for important intellectual content, gave final approval of the version to be published and agreed to be accountable for all aspects of the work.

Funding: Alison Galvani: National Institutes of Health (UO1-GM087719, 1R01AI151176-01); Burnett \& Stender Families Endowment; Notsew Orm Sands Foundation. Seyed Moghadas: CIHR (OV4 - 170643), COVID19 Rapid Research; Natural Sciences and Engineering Research Council of Canada); Canadian Foundation for Innovation.

Data sharing: Computational model with parameter values and data pertaining to the simulation study are freely available at: https://github. com/affans/covid19abm.jl

Accepted: Apr. 2, 2020

Correspondence to: Joanne Langley, Joanne.Langley@dal.ca 